\title{
UN ESTUDIO ACERCA DE LAS DIFERENCIAS DE GÉNERO EN LA RESOLUCIÓN DE PROBLEMAS CIENTÍFICOS ${ }^{1}$
}

\author{
POSTIGO, YOLANDA, PÉREZ ECHEVERRÍA, MARÍA DEL PUY y SANZ, ÁNGELES \\ Departamento de Psicología Básica. Universidad Autónoma de Madrid. 28049 Madrid.
}

\begin{abstract}
SUMMARY
This paper analyses the possible differences between Spanish secondary-school students (girls and boys) in solving mathematical and chemical tasks including relevant concepts and procedures from the curriculum in both areas. We study the effects of different variables on performances such as age and instruction level of subjects and the content and type of task. We also examine the relationship between the performance of boys and girls in chemical and mathematical tasks and their cognitive (Field Dependence/Independence) and attributional styles. We present the tasks to four groups: 13 and 15 year old adolescents, 17 year old adolescents without chemical training and 17 year old adolescents with chemical training. Within each group, half of the subjects were boys and half were girls. The results show differences in performance between boys and girls in solving mathematical and chemical tasks. In general, male performance is higher than female performance. However, these differences are not uniform: they change with age and type of instruction and with the content ant type of task. On the other hand, the data show no differences between boys and girls and cognitive style (Field Dependence/Independence). Likewise, the results reveal sex differences in attributional styles, although they are not correlated with the performance.
\end{abstract}

\section{INTRODUCCIÓN}

Una de las consideraciones en las que se hace especial hincapié en los nuevos currículos de enseñanza secundaria y bachillerato puestos en marcha en nuestro país es la atención especial a la diversidad de los alumnos. Dentro de esta diversidad, un aspecto importante es el género de nuestros estudiantes. A pesar de que la situación haya cambiado desde hace algunos años, parece que siguen existiendo diferencias entre los alumnos y las alumnas en el momento de elegir materias dentro del currículo y de decidir el campo profesional hacia el cual dirigir sus esfuerzos. Tradicionalmente los estudios y profesiones relacionados con la tecnología, las ciencias físicas y las matemáticas han sido consideradas como «masculinos», mientras que los estudios y profesiones relacionados con las ciencias sociales o el campo asistencial se han venido considerando actividades «femeninas».
Por otra parte, existen numerosos estudios que muestran diferencias de género en el rendimiento de tareas pertenecientes a áreas tales como las ciencias de la naturaleza y las matemáticas. Según algunos trabajos, desarrollados fundamentalmente en las décadas de los años sesenta y setenta, estas diferencias surgen en la adolescencia. Antes de esta etapa no existen diferencias o, si las hay, son a favor de las alumnas (Mayer, 1983). Sin embargo, las investigaciones más recientes muestran que estas diferencias son inestables y van disminuyendo de forma progresiva. Esta inestabilidad se puede deber a varias razones. En primer lugar, hablar de diferencias de género en ciencias o matemáticas es bastante vago y difuso, ya que las diferencias de tareas dentro de cada una de las disciplinas es lo suficientemente amplia como para encontrar una gran diversidad de rendimientos de cual- 
quier tipo. Cada uno de los trabajos atiende a tareas y problemas muy diferentes entre sí y, de la misma manera, las variables que intervienen son muy diferentes, por lo que las metodologías empleadas también cambian (Pérez Echeverría et al. 1992). Por lo tanto, resulta muy difícil establecer comparaciones entre estos distintos trabajos y resulta imposible (y seguramente poco útil e informativo) hablar de diferencias globales entre los dos géneros.

Así, por ejemplo, los trabajos centrados en el campo de las matemáticas han encontrando un patrón evolutivo, según el cual las diferencias de género son insignificantes durante los primeros años escolares y muestran un mayor rendimiento de las chicas. De forma progresiva, los chicos van mejorando su actuación en estas tareas, hasta que en la enseñanza secundaria se invierte el patrón y el rendimiento masculino es significativamente más alto que el femenino (Halpern, 1986; Hyde, Fennema y Lanon, 1990; Willms y Jacobsen, 1990). Por otra parte, los estudios llevados a cabo en el área de ciencias de la naturaleza han puesto de manifiesto que, en general, los chicos alcanzan pautas de rendimiento más altas que las chicas en tareas científicas; además estas diferencias se incrementan a medida que aumenta la edad (Howe y Doody, 1989; Levin, Sabar y Libman, 1991).

Pero, a su vez, estas diferencias dependen en buena medida, como afirmábamos antes, del área de conocimiento que estudiemos. Así, se ha encontrado que los alumnos tienen un mejor rendimiento que las alumnas en física (Bateson y Chatman, 1989) mientras que en biología la ventaja de los chicos es menos aparente o relativamente pequeña (Jiménez Alexaindre, 1990). En química, los estudios llevados a cabo muestran un dominio mayor de los chicos. No obstante, desconocemos si dentro de estos dominios (física, biología y química) existen a su vez diferencias de género en función del tipo de concepto implicado y de los subdominios de cada una de las áreas.

Es más, es posible que encontremos pautas variables no ya referentes a un área o subárea de conocimiento, sino en relación con las características de la tarea y el tipo de habilidades que exigen poner en juego. Así, las chicas muestran un dominio más alto de las habilidades computacionales (Hyde, Fennema y Lanon, 1990), mientras que los chicos, a partir de los catorce años, suelen resolver mejor los problemas (Hyde, Fennema y Lanon, 1990; Pérez Echeverría et al., 1992). Es decir, estos trabajos mostrarían que las chicas rinden más en tareas rutinarias que exigen poner en marcha procedimientos sobreaprendidos que en tareas en las que tienen que aplicar estos conocimientos a situaciones diferentes a las que las han aprendido. Uno de los contenidos en los que aparecen estas pautas que acabamos de reseñar es el campo relacionado con nociones proporcionales (Ethington, 1990; Pattison y Grieve, 1984). No obstante, la facilidad o dificultad de la tarea puede también mediar en estas diferencias. Por ejemplo, Karplus, Pulos y Stage (1983) encontraron que, a medida que aumentaba la dificultad de cómputo, aparecían mayores diferencias de las chicas respecto a los chicos, mientras que, en tareas de poca dificultad, el rendimiento era similar. Haciendo un rápido resumen, podríamos decir, por tanto, que las diferencias en el rendimiento entre los dos géneros dependen tanto del área de conocimiento como de características específicas de la tarea y del desarrollo de los sujetos.

Para complicar un poco más las cosas, como muestra un estudio realizado por la IEA (International Association for the Evaluation of Educational Achievement) entre 1983 y 1986, las diferencias de género varían en cada país analizado; por ejemplo, estas diferencias en países como Suecia son mínimas, sin embargo, en Australia, Canadá y Finlandia las diferencias encontradas están en torno al 0,40 o la sobrepasan (nuestro país no estaba incluido en este estudio). Es decir, las diferencias socioculturales parecen estar totalmente ligadas a estas diferencias de género, aunque normalmente no suele controlarse esta variable en las investigaciones. Podemos imaginar que estas diferencias entre diversos países serían mucho mayores si los países escogidos en la muestra estuvieran más lejanos socioculturalmente.

La presencia de diferencias socioculturales no parece invalidar la presencia de otros factores que pueden mediar en el diferente rendimiento en tareas matemáticas o científicas. Cierta cantidad de trabajos muestra la existencia de diferencias de género en relación con el estilo cognitivo dependencia-independencia de campo (Witkin y Goodenough, 1981). La dependencia-independencia de campo ha sido definida como la capacidad para procesar la información con alto grado de autonomía con respecto al campo perceptivo externo, de tal manera que los sujetos independientes de campo tienen una gran facilidad para separar los detalles de una configuración global. Aunque también hace referencia a otro tipo de factores, los trabajos sobre dependencia e independencia de campo parecen mostrar que los sujetos independientes de campo son más «analíticos» y los dependientes de campo más «sintéticos», lo cual influiría obviamente en su forma de procesar la información y en la manera de resolver problemas. Tradicionalmente se ha afirmado que los chicos muestran una mayor tendencia a la independencia de campo que las chicas (Witkin y Goodenough, 1981). No obstante, ni esta afirmación ni la relación de este constructo con el rendimiento académico están claras. Algunos estudios señalan, por ejemplo, que los sujetos independientes de campo son más efectivos en aquellos problemas de química que implican razonamiento proporcional (Falls y Voss, 1985) mientras que otros estudios no encontraron una relación significativa entre este constructo y el rendimiento en problemas de química (Chandran et al., 1987).

De la misma manera, las diferencias de género en distintos trabajos se han intentado explicar mediante los estilos atributivos. Según estos trabajos, las chicas tenderían a atribuir sus éxitos a factores externos como la suerte con más frecuencia que los chicos (Bar-Tal y Frieze, 1976), mientras que en situaciones de fracaso aludirían a la falta de habilidad ignorando el factor esfuerzo también con más frecuencia que sus compañe- 
ros de genero masculino (Wiegers y Frieze, 1977). Además, diversos estudios (Pérez Echeverría et al., 1992) han mostrado que existe cierta relación entre los estilos atributivos y el rendimiento académico pero muy pocos de ellos analizan este constructo en relación con las diferencias de género.

En el trabajo que estamos presentando nos interesa analizar las diferencias entre los dos géneros en el rendimiento en algunos problemas de química y de proporción. No obstante, en la medida que estas diferencias parecen depender, por un lado, de factores propios del sujeto, como la edad, cantidad y tipo de instrucción recibida, nos interesa analizar si estas posibles diferencias permanecen estables o cambian en función de la edad, curso y grado de instrucción en ciencias. Por otro lado, como hemos visto, también las diferencias están relacionadas con el tipo de tarea, por lo cual otro de nuestros objetivos es estudiar cómo influyen las características de los problemas en las posibles diferencias entre los dos géneros. Por último, nos interesa estudiar la relación entre el estilo cognitivo y el estilo atributivo en la medida en que podrían contribuir a explicar algunas de las diferencias de rendimiento entre los dos géneros en el caso de que las hubiera.

\section{MÉTODO}

\section{Sujetos}

La muestra utilizada estaba compuesta por 80 estudiantes, pertenecientes a cuatro grupos diferentes en función de su grado y tipo de escolaridad. Cada grupo estaba formado por 10 chicas y 10 chicos. Los distintos grupos estaban formados por estudiantes de $7^{\circ}$ de EGB (12-13 años de edad), $1^{\circ}$ de BUP (14-15 años de edad), $3^{\circ}$ de BUP sin instrucción en química (16-17 años de edad) y $3^{\circ}$ de BUP con instrucción en química (16-17 años de edad). Los adolescentes de $7^{\circ}$ de EGB eran alumnos de un colegio público de un barrio obrero de Madrid. A su vez los estudiantes de $1^{\circ}$ de BUP y $3^{\circ}$ de BUP cursaban sus estudios en otro barrio obrero de Madrid. La extracción social de todos los sujetos era media-baja. Por tanto, los grupos diferían entre sí: en la edad, en el curso y en su grado de instrucción en ciencias.

\section{Tareas}

Se utilizaron nueve tareas grupales de las cuales seis planteaban diversas tareas de matemáticas y química. Las otras tres tareas consistían en pruebas que evaluaban el estilo atributivo y el estilo cognitivo dependenciaindependencia de campo de los alumnos.

Las tareas de matemáticas y química hacían referencia tanto a problemas de proporcionalidad como a problemas sobre conservación de la sustancia y sobre la naturaleza corpuscular de la materia. Para evaluar el rendimiento en tareas de proporcionalidad se diseñaron tres cuestionarios. De estos tres cuestionarios (Tabla I, para un resumen de las tareas utilizadas), uno de ellos era exclusivamente numérico $(\mathrm{C} 1)$. Es decir, su presentación era exclusivamente matemática sin que hubiese ninguna referencia a algún otro tipo de contenido. En los otros dos cuestionarios se presentaron tareas de proporcionalidad aplicadas a la química (concentración de disoluciones). En un caso las tareas se presentaron en un

Tabla I

Tareas matemáticas y químicas utilizadas en la investigación.

\begin{tabular}{|c|c|c|c|}
\hline ÁREA & $\begin{array}{l}\text { FORMATO } \\
\text { DE PRESENTACIÓN }\end{array}$ & TAREAS & CONTENIDO \\
\hline \multirow[t]{3}{*}{ QUÍMICA } & \multirow[t]{3}{*}{ PROPOSICIONAL } & \multirow[t]{3}{*}{ NO CUANTITATIVO } & $\begin{array}{l}\mathrm{C}_{4} \text { : CONSERVACIÓN } \\
\text { DE LA SUSTANCIA }\end{array}$ \\
\hline & & & $\begin{array}{l}\mathrm{C}_{5}: \text { NATURALEZA } \\
\text { CORPUSCULAR DE LA MATERIA }\end{array}$ \\
\hline & & & $\begin{array}{l}\mathrm{C}_{6}: \text { PROPIEDADES } \\
\text { DE LAS PARTÍCULAS }\end{array}$ \\
\hline MATEMÁTICAS & PROPOSICIONAL & $\begin{array}{l}\text { COMPUTACIÓN } \\
\text { DE PROPORCIONES }\end{array}$ & $\begin{array}{l}\mathrm{C}_{1} \text { : COMPUTACIÓN } \\
\text { NUMÉRICA }\end{array}$ \\
\hline \multirow{2}{*}{$\begin{array}{l}\text { MATEMÁTICAS } \\
+ \\
\text { QUÍMICA }\end{array}$} & PROPOSICIONAL & \multirow{2}{*}{$\begin{array}{l}\text { COMPUTACIÓN } \\
\text { DE PROPORCIONES }\end{array}$} & $\mathrm{C}_{2}$ : DISOLUCIONES \\
\hline & ANALÓGICO & & $\mathrm{C}_{3}$ : DISOLUCIONES \\
\hline
\end{tabular}


formato proposicional (C2) y en el otro fue analógico (C3). Expresado con otras palabras, los problemas de la tarea $\mathrm{C} 2$ fueron presentadas por medio de proposiciones verbales, mientras que la tarea $\mathrm{C} 3$, tanto la cantidad de disolvente como la de soluto, fue presentada mediante dibujos.

En el cuestionario exclusivamente numérico (C1) se pedía a las y los estudiantes que decidieran cuál entre dos razones presentadas era mayor o si ambas eran iguales. En las tareas proporcionales presentadas, bien de modo proposicional (C2), bien de modo analógico (C3), los problemas consistían en mezclar o disolver un compuesto químico (cloruro sódico en agua). La tarea de los estudiantes consistía en averiguar cuál de dos recipientes, en los que previamente se había disuelto cloruro sódico en agua, estaba más concentrado o si ambos estaban igual de concentrados. Como hemos visto antes, en el cuestionario $\mathrm{C} 2$, los problemas se presentaron mediante proposiciones y, en el cuestionario $\mathrm{C} 3$, los problemas se presentaron mediante dibujos.

Se diseñaron tres cuestionarios para evaluar el rendimiento no cuantitativo en química. Uno de ellos estaba formado por once ítems sobre la conservación de la sustancia en los cambios físico-químicos (C4). De forma más exacta, el cuestionario estaba formado por ítems que planteaban problemas sobre varios fenómenos químicos, concretamente sobre disoluciones, cambios de estado y reacciones químicas. Cada problema tenía cuatro opciones de respuesta entre las cuales los sujetos debían elegir una. Los dos cuestionarios restantes planteaban cuestiones sobre la naturaleza corpuscular de la materia. El primero (C5) estaba formado por ocho ítems en los que los alumnos debían explicar un fenómeno químico (C5), eligiendo entre cuatro opciones de respuestas. Se trataba de evaluar si los sujetos tenían una concepción microscópica de la materia o no por medio de problemas sobre cinco contenidos químicos diferentes: disoluciones, cambios de estado, reacciones, difusión de los gases y dilataciones. Por último, el cuestionario C6 planteaba treinta problemas sobre algunas de las propiedades de las partículas o postulados sobre la teoría corpuscular de la materia. Concretamente los problemas trataban sobre el mecanismo explicativo de los cambios fisicoquímicos, tamaño y movimiento de las partículas, conservación del número de partículas, conservación de la sustancia y distribución de las partículas.

Para evaluar las atribuciones causales sobre los éxitos y fracasos de los sujetos en el área de logros académicos, utilizamos el EMA-II (Escala Multimensional de Atribución) adaptado por Alonso Tapia, Mateos y Montero, (1986). Esta prueba mide las atribuciones de los éxitos y fracasos académicos, en general, por medio de las siete escalas siguientes:

- Escala 1: Atribución del fracaso a la suerte

- Escala 2: Atribución del éxito a la suerte

- Escala 3: Atribución del fracaso al esfuerzo

- Escala 4: Atribución del éxito al esfuerzo
- Escala 5: Atribución del fracaso a la habilidad

- Escala 6: Atribución del éxito a la habilidad

- Escala 7: Atribución del éxito a la tarea

En cada uno de los ítems de la tarea, los estudiantes evaluaban su grado de acuerdo con una serie de expresiones por medio de una escala tipo Likert con un rango de 0 a 4 , donde 0 significaba máximo desacuerdo con el contenido de la afirmación y 4 significaba máximo acuerdo.

Debido a que esta tarea que acabamos de describir sólo hace referencia a atribuciones académicas en general y estas atribuciones pueden variar en función de las distintas tareas escolares, diseñamos un cuestionario sobre atribución de logro referido específicamente a las tareas de proporcionalidad y de química utilizadas en esta investigación. Este autoinforme (SENT) tenía las mismas escalas que la prueba anterior. También de la misma forma que en el cuestionario anterior, los estudiantes debían evaluar su grado de acuerdo con una serie de afirmaciones en una escala con un rango de 0 a 4 . Señalar 0 significaba que el estudiante no había tenido a lo largo de la tarea el pensamiento descrito en un determinado «ítem», mientras que 4 significaba que ese pensamiento se le había presentado con mucha frecuencia. Por último se utilizó el test de figuras enmascaradas, forma colectiva (Witkin, Oltman, Raskin y Karp, 1987) para evaluar el estilo cognitivo dependencia-independencia de campo.

\section{Procedimiento}

Los cuestionarios se aplicaron a los diferentes grupos en su colegio. Las pruebas se aplicaron en dos sesiones. El orden de aplicación de las pruebas se contrabalanceó aunque no de forma completa. De esta manera se presentaba siempre en primer lugar el EMA-II y, en último lugar, el autoinforme diseñado para evaluar las atribuciones de los sujetos sobre los éxitos y fracasos en las tareas presentadas de matemáticas y química. Este autoinforme se presentó en último lugar porque hacía referencia a las tareas que los sujetos acababan de realizar. El EMA se presentó en primer lugar con el objeto de separar los dos cuestionarios de atribución lo más posible y al mismo tiempo con el fin de que no interfiriera en la realización de las pruebas de matemáticas y química. Cada sesión duraba alrededor de hora y media y se concedía a los estudiantes un descanso de media hora aproximadamente entre ellas. Por tanto, el tiempo total de duración de las pruebas fue de alrededor de tres horas en cada uno de los grupos.

\section{Diseño}

Se utilizó un diseño 4 × 2 × 6, siendo los dos primeros factores intergrupo y el tercer factor intragrupo. El primer factor corresponde a los grupos $\left(7^{\circ}\right.$ de EGB, $1^{\circ}$ de BUP, $3^{\circ}$ de BUP sin formación en química y $3^{\circ}$ de BUP con formación en química); el segundo grupo, al 
Figura 1

Media de respuestas correctas para la interacción: género por grupo.

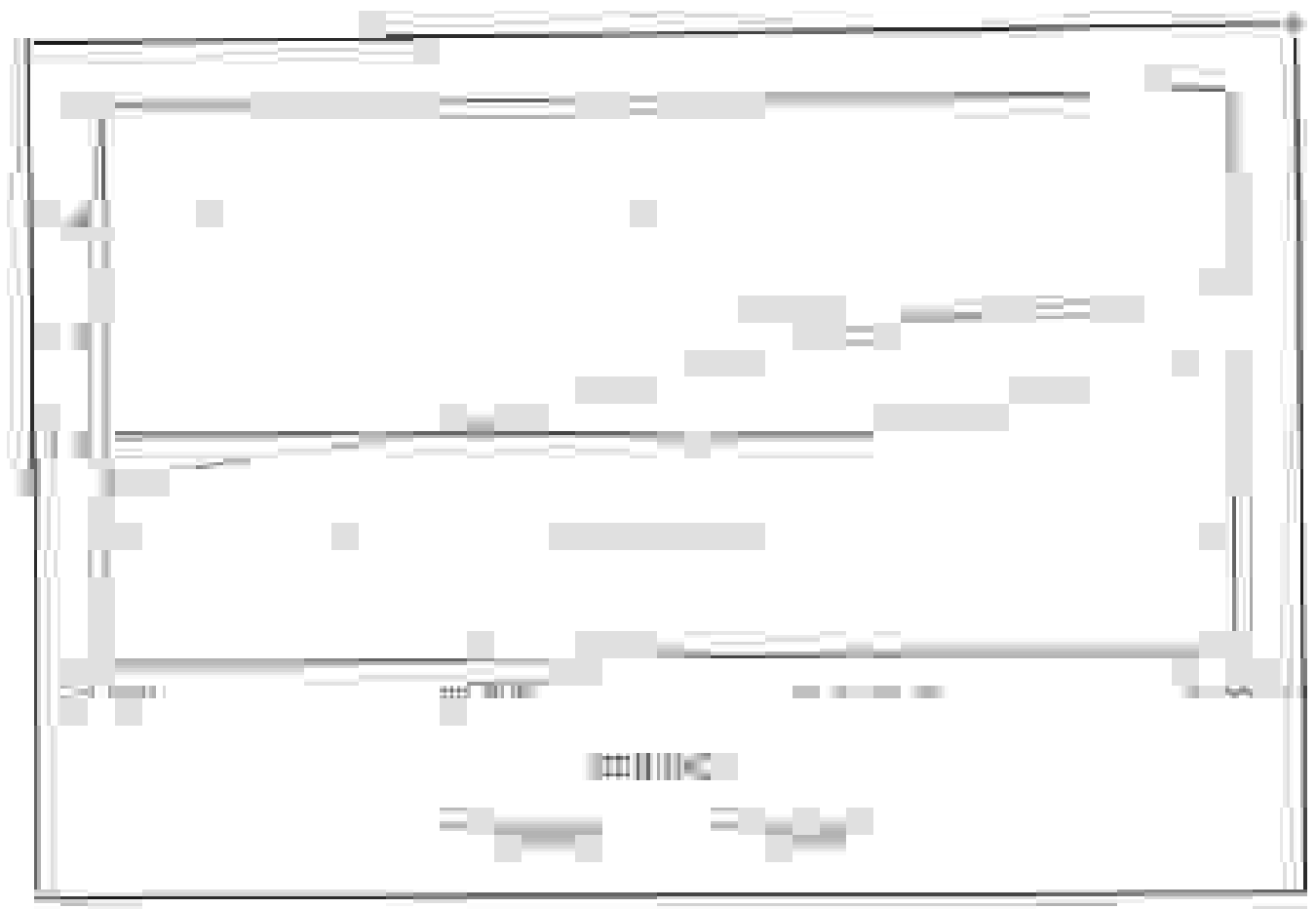

género de los estudiantes (masculino, femenino); y el tercero, a las tareas de matemáticas y química (proporción numérica, concentración de disoluciones proposicional y analógico, conservación de la sustancia, naturaleza corpuscular de la materia y propiedades de las partículas). Además se utilizó un diseño 4 (grupos) x 2 (géneros) para cada escala de atribución y para el GEFT.

\section{Criterios de corrección y análisis}

En las pruebas de matemáticas y química se analizó el número de aciertos en las tareas presentadas. En las escalas sobre atribuciones de los éxitos y fracasos se analizó el grado de acuerdo de los sujetos (0-4). En el GEFT analizamos el número total de las formas simples trazadas correctamente tal y como marca el manual (0-18). El criterio para establecer el estilo cognitivo dependencia-independencia de campo dependió de las medias de los propios grupos de referencia. Realizamos un ANOVA (análisis de varianza) para cada uno de los diseños descritos, lo cual nos permitiría establecer si existían o no diferencias significativas estadísticamente (no debidas al azar) dependientes de las variables analizadas. Además llevamos a cabo un ANCOVA (análisis de covarianza) 4 × 2 × 6 con cada una de las escalas como covariable para determinar si existía relación entre las escalas atributivas, el estilo cognitivo y las diferencias encontradas en el rendimiento de los estudiantes en las tareas de matemáticas y química.

\section{RESULTADOS}

\section{Rendimiento en la solución de problemas}

El análisis de varianza mostró un efecto significativo de la variable «género». Este resultado indica que existen diferencias significativas estadísticamente entre el rendimiento de las alumnas y de los alumnos en estas tareas. Los alumnos $($ media $=0,496)$ tienen un rendimiento global más alto que el de las alumnas (media $=0,438$ ). No obstante, este resultado puede matizarse en un doble sentido. En primer lugar, el número de respuestas correctas dadas tanto por las chicas como por los chicos fue algo inferior al 50\%. Es decir, el rendimiento global no fue muy alto y ello contribuye a que la diferencia entre los dos géneros sea significativa, aunque la diferencia general sea relativamente moderada. En segundo lugar, estas diferencias no aparecen en todas las tareas ni en todas las edades. 


\section{INVESTIGACIÓN DIDÁCTICA}

Así, el ANOVA (análisis de varianza) reveló la presencia de efectos significativos debidos a la interacción «género x grupo» Este resultado indica que las diferencias en el rendimiento entre las estudiantes y los estudiantes están mediadas por la edad de los sujetos y por el tipo de instrucción que han recibido. Los resultados obtenidos en esta interacción pueden observarse en la figura 1 .

Se encontraron diferencias significativas entre los dos géneros en los dos grupos mayores ( $3^{\circ}$ de BUP). El rendimiento de los alumnos de estos cursos fue de forma general más alto que el de las alumnas. En cambio, en el grupo de $1^{\circ}$ de BUP hubo un rendimiento global similar entre alumnas y alumnos en la resolución de tareas de proporcionalidad y químicas, mientras que las diferencias entre las alumnas y los alumnos más jóvenes $7^{\circ} \mathrm{de}$ EGB estuvieron muy próximas al nivel de significación. Como puede verse en la figura 1 , el rendimiento de las estudiantes (media $=0,412$ ) fue claramente superior al de sus compañeros masculinos $($ media $=0,325)$.

En definitiva, estos resultados indican que sólo a partir de la adolescencia hay un rendimiento más alto de los alumnos frente a las alumnas. Antes de esta edad no existen diferencias o estas diferencias son a favor de las mujeres. Sin embargo, las diferencias de género encontradas además de no ser homogéneas a todas las edades no se distribuyen por igual en todas las tareas. En efecto, el análisis de varianza encontró la interacción «género $\mathrm{x}$ tarea» significativa. Como muestra la figura 2, el rendimiento de los alumnos fue mayor que el de las alumnas en tareas proporcionales aplicadas a la química (tanto para las tareas presentadas con formato proposicional como analógico) y en las tareas centradas en la naturaleza corpuscular de la materia (continuidad-discontinuidad).

Por tanto, los resultados señalan que las diferencias de género en el rendimiento en problemas matemáticos y científicos se ven afectados en buena medida por el tipo de tarea planteado. Además encontramos que, ante tareas cuantitativas isomórficas, el rendimiento de los estudiantes varía en función de que se plantee una tarea en términos exclusivamente numéricos o aplicada al campo de la química (no se encontraron diferencias entre las alumnas y los alumnos en el primer tipo de tarea pero sí en la segunda). Sin embargo, tampoco estos resultados son iguales para todos los grupos de edad e instrucción, por lo que es necesario comparar el rendimiento de los alumnos y alumnas de los distintos grupos para cada tipo de tarea.

$\mathrm{El}$ análisis de varianza de estas tres variables, «género $\mathrm{x}$ grupo $\mathrm{x}$ tarea», mostró que el rendimiento de las alumnas fue mayor que el de los alumnos en $7^{\circ}$ de EGB en tareas proporcionales numéricas, mientras que no había diferencias significativas en $1^{\circ}$ de BUP. En el grupo de $3^{\circ}$ de BUP sin instrucción en química se encontraron diferencias a favor de los estudiantes varones en las tareas cuantitativas tanto aplicadas a un contenido químico $(\mathrm{C} 2$

Figura 2

Media de respuestas correctas para la interacción: género por contenido.

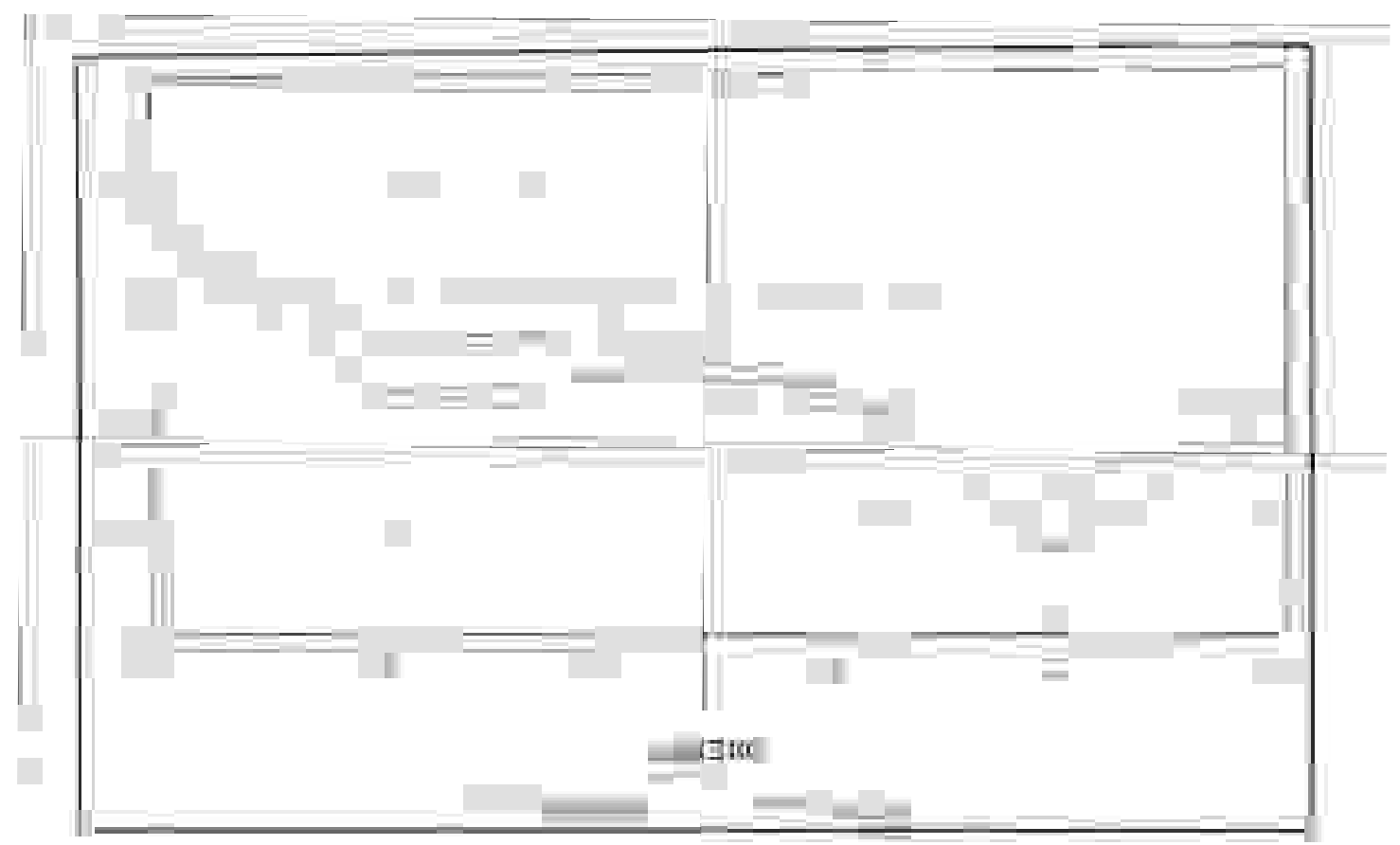


Figura 3

Media de respuestas correctas para la interacción: género por contenido para el grupo de $3^{\circ}$ sin formación en química.

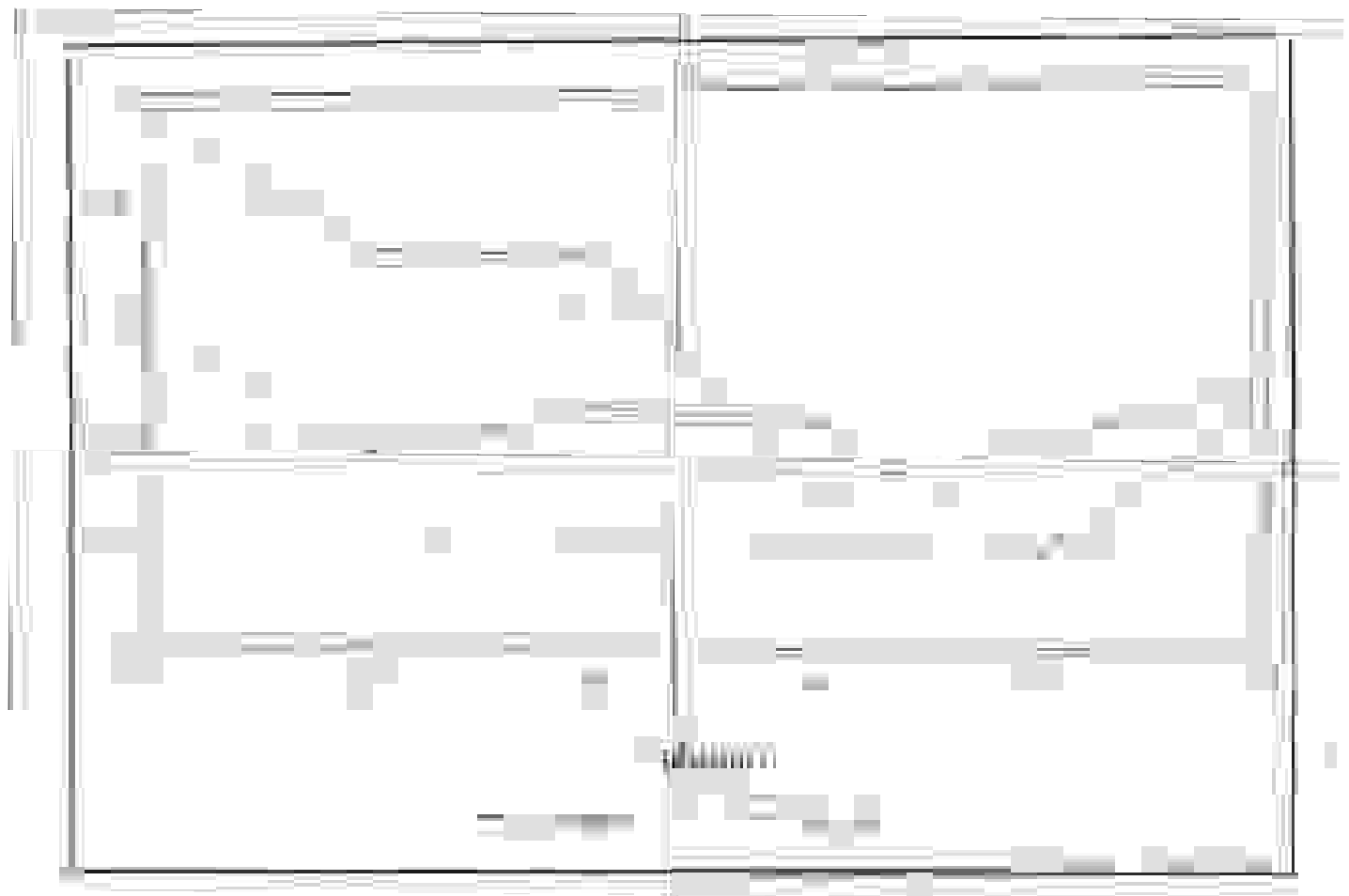

y C3) como exclusivamente numéricas (C1). En cambio, en el grupo de $3^{\circ}$ de BUP con instrucción en química los alumnos rendían más que las alumnas en tareas químicas, no cuantitativas. Concretamente las diferencias se produjeron en la tarea sobre conservación de la sustancia después de haberse producido diversos cambios físicoquímicos (C4) y la tarea sobre las propiedades de las partículas (C6).

Al comparar los grupos de $3^{\circ}$ de BUP (Fig. 3 y 4) podemos observar que la instrucción afecta de manera diferente a las alumnas que a los alumnos, de tal modo que las alumnas mejoran significativamente en razonamiento proporcional tanto aplicado a contenidos químicos como en tareas exclusivamente numéricas. En cambio, la mejora de los alumnos es de tipo más bien cualitativo o no cuantitativo, ya que en el grupo de $3^{\circ} \mathrm{de}$ BUP con entrenamiento en ciencias se encuentran diferencias a favor de los estudiantes varones en las tareas de conservación de la sustancia y propiedades de las partículas.

\section{Estilos cognitivos y estilos atributivos}

\section{Estilos cognitivos}

El ANOVA (análisis de varianza) no mostró diferencias significativas entre las alumnas y los alumnos en el estilo cognitivo dependencia-independencia de campo (la media para el grupo de alumnas fue de 12,23 y la media para el grupo de alumnos fue de 12,53). Asimismo el análisis de varianza no halló diferencias significativas entre los estudiantes y las estudiantes en los distintos grupos. Sin embargo, en el grupo de $3^{\circ}$ de BUP con formación en química tanto en las alumnas como en los alumnos, pero en mayor medida en estos últimos, se encontró una tendencia mayor hacia la independencia de campo (por ejemplo, la media del grupo de alumnos de tercero de BUP con formación en química fue de 14,83 frente al resto de los grupos que no sobrepasaron la media de $11,90)$.

\section{Estilos atributivos}

En cuanto a las atribuciones para los logros académicos generales, el análisis mostró diferencias de género. Así, las alumnas suelen atribuir, con más frecuencia que los alumnos, el éxito en tareas escolares, a la suerte -factor externo e inestable- (la media para las alumnas fue de 2,15 y la media de los alumnos fue de 1,81), mientras que atribuyen el fracaso a la falta de habilidad (factor interno y estable) con más frecuencia que sus compañeros del género masculino (la media para el grupo de alumnas fue de 3,03 y la media para el grupo de alumnos fue de 2,74). Esta tendencia, no obstante, se ve mediada por el grado y tipo de instrucción, siendo especialmente relevante en el caso de las alumnas más jóvenes $\left(7^{\circ}\right.$ de EGB) y en el 
Figura 4

Media de respuestas correctas para la interacción: género por contenido para el grupo de $3^{\circ}$ con formación en química.

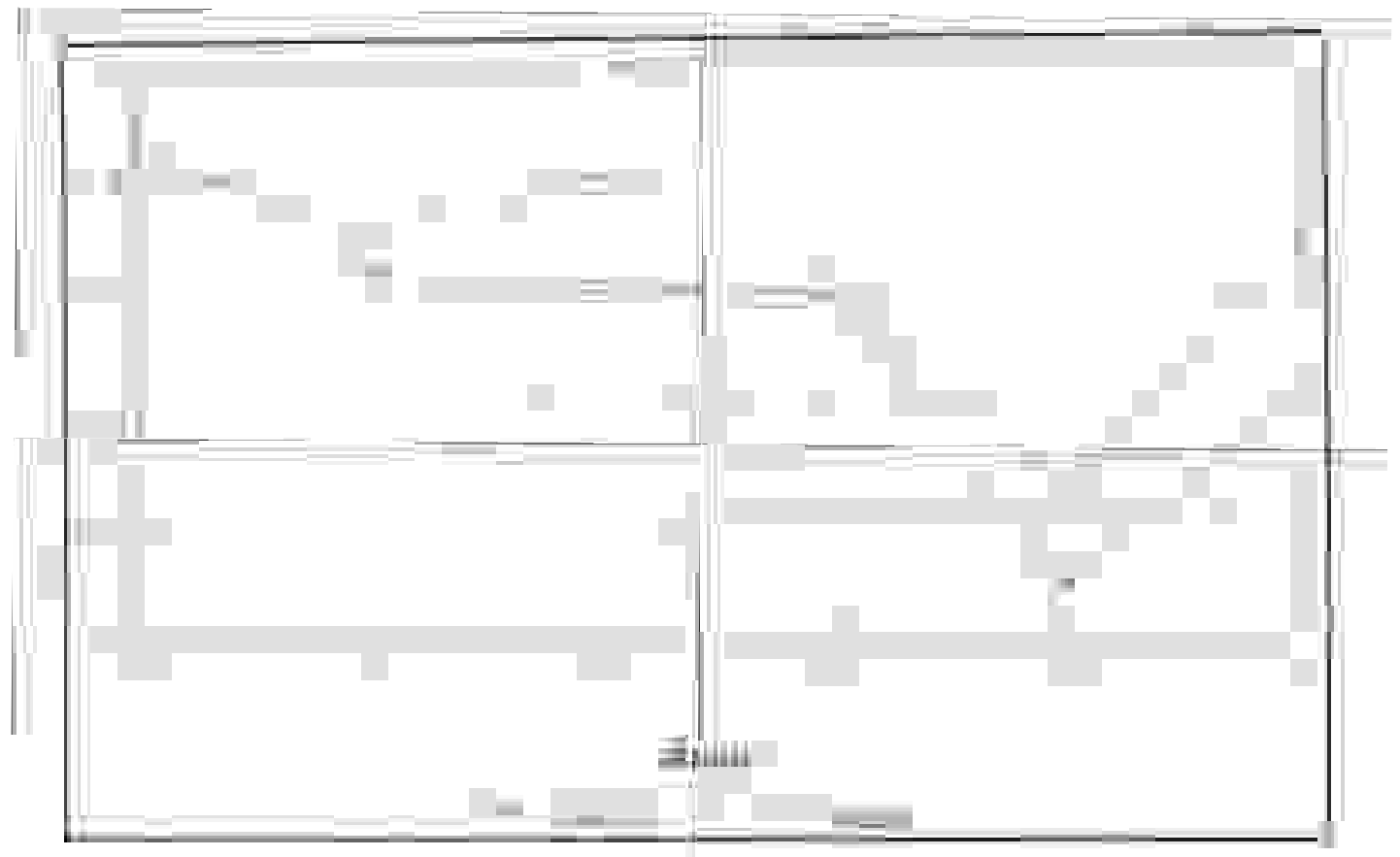

caso de las alumnas de $3^{\circ}$ de BUP sin formación en química.

Aparecen diferencias de género en la forma en que alumnas y alumnos atribuyen sus éxitos a la habilidad (la media de los alumnos fue de 1,44 y la media de las alumnas fue de 0,98 ) y a la facilidad de la tarea (la media de los alumnos fue de 2,04 y la de las alumnas fue de 1,45). A diferencia de las chicas, los alumnos tienden a atribuir su éxito a la habilidad y a la facilidad de la tarea más que a otros factores. No obstante, el análisis de covarianza muestra que este tipo de atribución no se correlaciona con el rendimiento en estas tareas.

\section{DISCUSIÓN Y CONCLUSIONES}

Nuestra primera conclusión podría ser que existen diferencias entre el rendimiento de las alumnas y de los alumnos en estas tareas de química y de proporcionalidad, ya que los alumnos muestran un rendimiento significativamente más alto que las alumnas. No obstante, es necesario matizar esta conclusión. En primer lugar, el número de respuestas correctas oscilaba entre el $40 \%$ y el $50 \%$ tanto en el caso de las alumnas como en el de los alumnos. Este resultado indica que, en conjunto, los sujetos respondieron bien a menos de la mitad de los problemas propuestos. Es posible que ese hecho haya contribuido a que estas diferencias sean significativas a pesar de que, en términos absolutos, la distancia entre ellas no sea muy alta. Por otro lado, estas diferencias están mediadas por la edad y la instrucción de las y los estudiantes y por las características de la tarea

Las diferencias entre los dos géneros dentro de cada uno de los grupos responden a un patrón encontrado en numerosas investigaciones sobre el rendimiento tanto en tareas matemáticas como de tipo científico (Halpern, 1986; Hyde, Fennema y Lanon, 1990; Mayer, 1983 -en el caso de las matemáticas-; y Howe y Doody, 1989; Levin, Sabar y Libman, 1991; Pozo, Gómez Crespo, Limón y Sanz, 1991 -en el caso de las ciencias naturales). Este patrón indica que el grado de estas diferencias aumenta conforme va aumentado la edad de las y los estudiantes. Así, nosotras encontramos que las únicas diferencias significativas se daban dentro de los dos grupos de $3^{\circ}$ de BUP. Como en los trabajos que hemos mencionado, nuestros resultados mostraban un rendimiento más alto de los varones respecto a las mujeres de la muestra. Sin embargo, como hemos visto, estas diferencias no se desarrollan del mismo modo para todas las tareas.

No obstante, a pesar de que las únicas diferencias significativas aparecían en $3^{\circ}$ de BUP, nos parece importante señalar que, en el grupo de menor edad ( $7^{\circ}$ de EGB), las disimilitudes en la actuación eran muy próximas al nivel 
de significación. En este grupo, las alumnas mostraban un rendimiento más alto que los alumnos. Este resultado también es concordante con los trabajos que hemos mencionado antes y que muestran que antes de los 14-15 años aproximadamente no existen divergencias entre la actuación de alumnas y alumnos o muestran un rendimiento más alto de las alumnas. Es posible que una muestra de estudiantes de menor edad con este tipo de tareas nos hubiera mostrado diferencias más altas. Pero, de la misma forma que en los grupos de $3^{\circ}$ de BUP, resulta necesario acudir al tipo de tarea para explicar estas diferencias.

Cuando revisamos la actuación en las diferentes tareas que hemos planteado, encontramos que las alumnas de $7^{\circ}$ de EGB daban un número de respuestas correctas significativamente más alto en la tarea de proporcionalidad presentada sin contenido químico. Es decir, las diferencias aparecían en la tarea más fácil si atendemos al número global de respuestas correctas (Pérez Echeverría, Postigo y Sanz, 1992, para un análisis sobre el efecto de la edad o instrucción y la tarea en el rendimiento con independencia del género). Este resultado coincide parcialmente con algunos trabajos realizados en el área de solución de problemas matemáticos (Bridgeman y Wendler, 1991; Ethington, 1990; Hyde, Fennema y Lannon, 1990). Estas investigaciones muestran que, en general, las chicas tienen un rendimiento más alto que los chicos en tareas que requieren habilidades computacionales o en problemas de tipo algebraico en edades comprendidas entre los 11 y 14 años aproximadamente. En los problemas encuadrados dentro de la tarea $\mathrm{C}$ 1, sólo se pedía a las adolescentes que computaran las posibles diferencias entre dos razones presentadas con un formato algebraico sin que se mencionara ningún referente al contenido de estas fracciones.

Sin embargo, estos resultados están en desacuerdo con los trabajos de Ethington (1990) y Pattison y Grieve (1984), que muestran un rendimiento mayor de los alumnos respecto a las alumnas en tareas en las que subyace el conocimiento y la utilización de la noción de proporción. No obstante, las investigaciones realizadas por estos autores difieren en algunos aspectos importantes de nuestro trabajo. El trabajo de Ethington es un avance de los resultados de una evaluación de las diferencias de género en distinto tipo de tareas matemáticas en diversos países. Aunque, en general, la actuación de los estudiantes masculinos en problemas de proporcionalidad era más alta que la de sus compañeras, estas diferencias variaban de un país a otro. Además, el autor no describe los problemas utilizados en su investigación, por lo cual resulta muy difícil establecer comparaciones. Por otro lado, en el estudio de Pattison y Grieve, los problemas de proporcionalidad forman parte de un trabajo más amplio cuyo objetivo principal es analizar las diferencias en resolución de tareas matemáticas en las que están implícitos factores espaciales. Además estos problemas están presentados mediante palabras, mientras que la tarea que estamos analizando estaba presentada en un formato algebraico. Es posible que los resultados de Pattison y Grieve estén mediados por factores diferentes a los que han influido en nuestra tarea.
En el grupo de alumnas y alumnos de $1^{\circ}$ de BUP no apareció ningún tipo de diferencias significativas en las distintas tareas presentadas. Sin embargo, tanto en el grupo de $3^{\circ}$ de BUP con instrucción específica en conceptos y métodos químicos como en el grupo de $3^{\circ}$ que no tenía esta instrucción, apareció este tipo de diferencias. Resulta curioso observar que, en este último grupo, los resultados indican un rendimiento significativamente más alto de los alumnos en las tres tareas proporcionales ( $\mathrm{C} 1, \mathrm{C} 2$ y C3), mientras que en el grupo con instrucción en química las diferencias se centran en el cuestionario sobre conservación de la sustancia después de haber sido sometida a cambios fisicoquímicos (C4) y en el cuestionario sobre propiedades de las partículas (C6). Por tanto, podríamos decir que, mientras que en el primer grupo (sin instrucción específica en química), las diferencias se centran en los problemas más cuantitativos, en el segundo grupo (con instrucción específica), los resultados indican que estas discrepancias se refieren a los problemas más cualitativos.

La pauta de respuestas en las y los estudiantes de $3^{\circ}$ de BUP con formación en química es similar a la encontrada en otras investigaciones que muestran que el rendimiento de las alumnas en tareas conceptuales de química es menor que el de sus compañeros masculinos (Howe y Doody, 1989; Levin, Sabar y Libman, 1991). No obstante, cuando comparamos las medias de respuestas correctas dadas en los dos grupos de $3^{\circ}$ de BUP, encontramos que las diferencias entre los dos grupos de chicos son menores que entre los dos grupos de chicas, aunque en ambos casos son significativas (Fig. 1). Por tanto, parece que la instrucción específica tiene claras consecuencias en el rendimiento. Las alumnas con instrucción específica en química mejoran significativamente respecto a las alumnas que han elegido otras opciones. Estas diferencias son más patentes en el caso de las tareas cuantitativas pero están presentes en todas las tareas. Es necesario tener en cuenta la diferencia en atribución entre estos dos grupos que puede haber contribuido a una selección previa de las integrantes de cada una de las opciones. Así, las alumnas de $3^{\circ}$ de BUP sin formación en química atribuyen más los fracasos escolares a la falta de habilidad que sus compañeros masculinos. Este tipo de atribución puede ocasionar una falta de persistencia en el trabajo dentro de aquellas materias en las que se considera la habilidad como el factor más importante (en general, matemáticas y ciencias) y, por tanto, a la larga se derivaría en un rendimiento menor ante este tipo de tareas. Por otro lado, el hecho de que no existan estas diferencias atribucionales en el grupo de $3^{\circ}$ con formación en química podría corroborar este hecho en el sentido de que aquellas alumnas que consideran que tienen una baja habilidad para estas materias (factor interno y estable) no eligen una opción en sus estudios que pueda poner de manifiesto las creencias sobre sus propias habilidades.

En resumen, parecen existir diferencias en el rendimiento de las alumnas y alumnos españoles en estas materias, pero, como muestran también diversos trabajos llevados a cabo desde la psicología de la instrucción, estas diferencias están claramente mediadas por la edad, la ins- 
trucción y el contenido y tipo de tarea analizado (Gómez Crespo, et al., 1995). Los resultados obtenidos con el cuestionario GEFT (prueba de las figuras enmascaradas, versión grupal), muestran que no existen diferencias significativas entre los dos géneros en cuanto a su dependencia-independencia de campo así como entre los distintos grupos de la muestra. Estos resultados nos indican que no podemos utilizar este factor para explicar las diferencias en el rendimiento encontradas en estas tareas químicas y proporcionales. No obstante, a pesar de la ausencia de diferencias significativas, en nuestros resultados parece haber una tendencia general que muestra que las y los componentes del grupo de $3^{\circ}$ de BUP con formación en química muestran una mayor independencia de campo. Expresado con otras palabras, la instrucción en química parece estar relacionada con una mayor independencia de campo, aunque, como hemos dicho, este resultado no es significativo estadísticamente. Esta tendencia es aún mayor en los alumnos masculinos de este grupo tanto si la comparamos con los estudiantes de otros grupos como si la comparamos con sus compañeras de curso.

Por otro lado, podríamos hipotetizar que la ausencia de diferencias significativas entre los dos géneros, en lo referente a este factor, puede ser motivada por los cambios sociales y culturales y por la incorporación masiva de la mujer a la educación. Como señalan distintos autores (Van Leuwen, 1978; Witkin, Oltman, Raskin, Karp, 1987), las diferencias en la DIC están reflejando diferencias en las pautas de socialización entre hombres y mujeres. Nuestros resultados indican la presencia de este tipo de diferencias pero su magnitud no es suficiente para que sean significativas. Por tanto, podrían estar reflejando los cambios sociales a los que aludíamos antes. Además, como indican Witkin y otros (1975, 1977), las diferencias entre los dos géneros suelen ser leves si las comparamos con las diferencias entre individuos de un mismo género.

Estas hipótesis que acabamos de aventurar con respecto a las diferencias de género en el estilo cognitivo dependencia-independencia de campo podrían también ser útiles para explicar el hecho de que los estilos atributivos no medien en el rendimiento alcanzado en las tareas propuestas. No obstante, a pesar de que los estilos atributivos no constituyen una variable mediadora, los resultados muestran, como hemos visto antes, diferencias importantes entre las alumnas y los alumnos.

En primer lugar, encontramos que las alumnas suelen, de forma significativa, atribuir más frecuentemente que los alumnos el éxito, en tareas escolares, a la suerte (factor externo e inestable) mientras que atribuyen el fracaso a la falta de habilidad (factor interno y estable). Este tipo de patrón encontrado en las alumnas del género femenino coincide con el tipo de atribución denominado «indefensión aprendida» (Abramson, Seligman y Teasdale, 1978). Como han señalado numerosos autores (Forsyth, 1986; Powers y Wagner, 1984; Weiner, 1986), la forma en que los alumnos perciben su éxito o fracaso en el trabajo escolar puede tener un impacto significativo sobre las expectativas para su futura actuación, su estado de ánimo y su posterior rendimiento académico. En este sentido Rogers (1987) afirma que las personas no responden sólo al éxito o al fracaso, como tales. Responden a sus éxitos y fracasos a la luz de la interpretación que hacen de ellos, la cual, estará en gran medida determinada por las causas que, según ellos, son responsables del éxito o del fracaso. En este sentido, las alumnas cuyo rendimiento hemos examinado podrían estar mostrando un tipo de atribución que contribuiría muy poco a su éxito escolar en la medida que achacan los éxitos a factores externos como la suerte y los fracasos a su falta de competencia.

Esta tendencia en la atribución femenina parece ser especialmente relevante en el caso de las alumnas más jóvenes y en el caso de las alumnas de $3^{\circ}$ de BUP sin formación específica en química. Las alumnas de estos grupos atribuyen sus fracasos a la falta de habilidad con más frecuencia que sus compañeros y con más frecuencia que las alumnas de $1^{\circ}$ de BUP y de $3^{\circ}$ con formación en química. Como veíamos en el apartado anterior, es probable que esta atribución haya contribuido a una selección previa de los grupos. Así, parece razonable que aquellas estudiantes que no creen tener la suficiente competencia para enfrentarse a disciplinas en las que tradicionalmente se considera que la capacidad intelectual juega un papel mayor que en otras materias (por ejemplo, matemáticas y ciencias) opten, en el sistema educativo, por opciones en las que no tienen que poner a prueba, desde su propio punto de vista esta habilidad (por ejemplo, filosofía y letras, historia, filología).

En el cuestionario que diseñamos específicamente para analizar el tipo de causas atribuidas por las alumnas y alumnos a su rendimiento en las tareas experimentales, este patrón que acabamos de describir se confirma parcialmente. A diferencia del caso anterior no encontramos un patrón de indefensión aprendida en las alumnas. No obstante, los resultados indican la presencia de diferencias en las atribuciones entre las alumnas y los alumnos. Los estudiantes masculinos atribuyen con más frecuencia su posible éxito en estas tareas tanto a su habilidad como a la facilidad de la tarea. Es decir atribuyen sus éxitos a factores estables, aunque, en el primer caso, se trate de un factor interno y, en el segundo, de un factor externo. Estos resultados estarían en consonancia con los encontrados por Ryckman y Peckham (1987) dentro del área de matemáticas en general. En este sentido, encontramos que los alumnos tienen una mayor confianza en su habilidad para resolver estas tareas matemáticas y científicas que sus compañeras.

Por tanto, resumiendo, podríamos afirmar que, aunque no hemos encontrado que los estilos atributivos sean una variable mediadora del rendimiento en las tareas experimentales que hemos utilizado, nuestros resultados muestran la presencia de algunas diferencias en las atribuciones de alumnas y alumnos tanto de tipo general como referidas específicamente a las tareas realizadas. Al interpretar estos resultados es necesario tener también en cuenta que tanto las alumnas como los alumnos tendían a utilizar más a menudo los valores centrales de las escalas en ambos cuestionarios que los valores más extremos. 
Este hecho puede haber contribuido a que encontremos un menor grado de diferencias entre ambos géneros.

Para finalizar, podríamos decir que nuestros resultados muestran diferencias entre las alumnas y los alumnos en distintas tareas químicas y matemáticas. La naturaleza de estas diferencias es muy compleja y es razonable pensar que en ella intervienen numerosos factores tanto de tipo psicológico como sociológico, o cultural, o de otros órdenes. En nuestra investigación hemos podido observar que estas diferencias no son estables sino que están interrelacionadas con factores de la tarea (contenido) y con factores de la persona distintos del género al que pertenece (edad, nivel y tipo de instrucción). El tipo de atribución del logro académico es una variable que puede estar influyendo en estas diferencias aunque nuestros resultados no nos permiten afirmar que actúa como variable mediadora. No obstante, podemos esperar que también estén interactuando otras variables relacionadas con el mundo académico y social como pueden ser las expectativas de éxito o fracaso, el interés por las tareas realizadas, etc.
A nuestro juicio, uno de los resultados más interesantes que hemos obtenido ha sido el papel de la instrucción en estas diferencias. Aunque hemos encontrado diferencias favorables a los alumnos en los dos grupos de edad superior $\left(3^{\circ}\right.$ de BUP con y sin formación específica en química), la ganancia relativa obtenida por las alumnas tras la instrucción era más alta que la de sus compañeros masculinos. Este resultado parece indicar que, independientemente del origen de las diferencias entre los dos géneros, la instrucción es una poderosa herramienta que puede contribuir a un rendimiento más equivalente entre ambos.

\section{NOTAS}

${ }^{1}$ Este trabajo ha sido posible gracias a la concesión de una ayuda del Instituto de la Mujer a un proyecto de investigación dirigido por la tercera autora $\mathrm{y}$ a un proyecto DIGICYT (PB 94-0188) dirigido por J.I. Pozo.

\section{REFERENCIAS BIBLIOGRÁFICAS}

ABRAMSON, L.Y., SELIGMAN, M.E.P. y TEASDALE, J. (1978). Learned helplessness in humans: critique and reformulation. Journal of Abnormal Psychology, 87, p. 49-74.

BAR-TAL, D. y FRIEZE, I. H. (1976). Attribution of success and failure for actors and observers. Journal of Research in Personality, 10, pp. 256-265.

BATESON, D. J. y PARSONS-CHATMAN, S. (1989). Sexrelated differences in science achievement: a possible testing artifact. International Journal Science Education. 11(4), pp. 371-385.

BRIDGEMAN, B. y WENDLER, C. (1991). Gender differences in predictors of college mathematics performance and in college mathematics course grades. Journal of Educational Psychology, 83(2), pp. 275-284.

CHANDRAN, S., TREAGUST, D.F. y TOBIN, K. (1987). The role of cognitive factors in chemistry achievement. Journal of Research in Science Teaching, 24(2), pp. 145-160.

ETHINGTON, C. A. (1990). Gender differences in mathematics: an international perspective. Journal for Research in Mathematics Education, 21(1).
FALLS, T.H. y VOSS, B. (1985). The ability of high school chemistry students to solve computational problems requiring proportional reasonig as affected by items in task variables. Annual meeting of the National Association for Research in Science Teaching, 58, 15-18 de abril. French LickSprings, IN.

FORSYTH, D.R. (1986). An attributional analysis of students' reactions to success and failure, en Feldman, R.S. (ed.) Social Psychology of Education. Nueva York: Cambridge University Press.

GÓMEZ CRESPO, M. A., POZO, J. I. y SANZ, A. (1995). Students' conceptions about conservation of matter. Science Education.

HALPERN, D. F. (1986). Sex differences in cognitive abilities. Hillsdale, Nueva Jersey: LEA.

HOWE, A.C. y DOODY, W. (1986). Spatial visualization and sexrelated differences in science achievement. Science Education, 73(6), pp. 703-709.

HYDE, J.S., FENNEMA, E. y LAMON, S.J. (1990). Gender differences in mathematics performance: a meta-analysis. Psychologica Bulletin, 107(2), pp. 139-155. 
JIMÉNEZ ALEXAINDRE, M.P. (1990). Los esquemas conceptuales sobre la selección natural: análisis y propuestas para un cambio conceptual. Madrid: Universidad Complutense de Madrid. Tesis doctoral, edición facsímil.

KARPLUS, R., PULOS, S. y STAGE, E.K. (1983). Proportional reasoning of early adolescents, en Lesh, R. y Landau, M. Acquisition of mathematics concepts and processes. Nueva York: Academic Press.

LEVIN, T., SABAR, N. y LIBMAN, Z. (1991). Achievements and attitudinal patterns of boys and girls in science. Journal of Research in Science Teaching, 28(4), pp. 315-328.

MAYER, R.E. (1983).Thinking, problem solving and cognition. W.H. Freeman and company. Trad. de Maldonado, A El futuro de la psicología cognitiva. Barcelona: Paidós.

PATTISON, P. y GRIEVE, N. (1984). Do spatial skills contribute to sex differences in different types of mathematical problems. Journal of Educational Psychology, 76(4), pp. 678-689.

POWERS, S. y WAGNER, M.J. (1984). Attributions for school achievement of middle school students. Journal of Early Adolescence, 4, pp. 215-222.

PÉREZ ECHEVERRÍA, M.P., POSTIGO, Y. y SANZ, A. (1992). Las alumnas y alumnos ante las ciencias y las matemáticas: influencia de los estilos cognitivos y atributivos. Madrid: Universidad Autónoma. Memoria de Investigación inédita.

POZO, J. I., GÓMEZ CRESPO, M.A; LIMÓN, M. y SANZ, A. (1991). Procesos cognitivos en la comprensión de la ciencia: ideas de los alumnos sobre la química. Madrid: Servicio de Publicaciones del MEC.

ROGERS, C. (1982). A social of psychology of schooling. Nueva York: Routledge and Kegan Paul. Trad. de Pérez
Echeverría, M.P. y Pozo, J.I., 1987. Psicología social de la enseñanza. Madrid: MEC-Visor.

RYCKMAN, D. y PECKMAN, P. (1987). Gender differences in attribution for success and failure. Journal of Early Adolescence. 7, pp. 47-63.

VAN LEUWEN, M.S. (1978). A cross-cultural examination of psychological differentation in males and females. International Journal of Psychology, 13, pp. 87-122.

WEINER, B. (1986). An attributional theory of motivation and emotion. Nueva York: SpringerVerlag.

WIEGERS, I. M. y FRIEZE, I. H. (1977). Gender, female traditionality, achievement level and cognitions of success and failure. Psychology of Women Quarterly, 2, pp. 125-137.

WILLMS, J.D. y JACOBSEN, S. (1990). Growth in mathematics skills during the intermediate years: sex differences and school effects. International Journal of Educational Research. 14, pp. 157-160.

WITKIN, H.A. y BERRY, J. W. (1975) Psychological differentation in crosscultural perspective. Journal of Cross Cultural Psychology, 6, pp. 4-87.

WITKIN, H.A. y GOODENOUGH, D.R. (1981). Cognitive styles: essence and origins. Nueva York: International Universities Press, Inc. Trad. de Sánchez López, P. Estilos cognitivos: naturaleza y orígenes. Madrid: Pirámide.

WITKIN, H.A., MOORE, C.A., GOODENOUH, D.R. y COX, P.W. (1977). Fielddependent and fieldindependent cognitive styles and their educational implications. Review of Educational Research. 47, pp. 1-64.

WITKIN, H.A., OLTMAN, P.K., RASKIN, E. y KARP, S.A. (1987). Test de figuras enmascaradas (forma colectiva GEFT). Madrid: TEA (2a. ed. revisada).

[Artículo recibido en octubre de 1995 y aceptado en diciembre de 1998.] 